\title{
QoS-Constrained Robust Beamforming in MISO Wiretap Channels with a Helper
}

\author{
Jing Huang and A. Lee Swindlehurst \\ Electrical Engineering and Computer Science \\ University of California, Irvine, CA 92697 \\ Email: \{jing.huang; swindle\}@uci.edu
}

\begin{abstract}
In this paper, we develop robust beamforming strategies for multiple-input single-output (MISO) wiretap channels with a helper. The channel state information (CSI) for the legitimate link is assumed to be available while the CSI for the eavesdropper's channel is imperfect, and is assumed to be norm-bounded by some known constant. We optimize the worstcase performance for the following problems: 1) minimizing the signal to interference-plus-noise ratio (SINR) at the eavesdropper while maintaining an SINR constraint at the intended receiver, 2) maximizing the SINR at the intended receiver while forcing an SINR constraint at the eavesdropper, and 3) minimizing the global transmit power while satisfying SINR constraints at both the legitimate user and the eavesdropper. Simulation examples show the advantages of the robust designs over the non-robust counterparts.
\end{abstract}

\section{INTRODUCTION}

Secure transmission is traditionally considered as an issue addressed above the physical (PHY) layer, and conventional approaches for ensuring confidentially are usually based on cryptographic methods. However, the broadcast nature of wireless transmissions and the dynamic topology of mobile networks may introduce significant challenges to secret key transmission and management. Therefore, there has recently been considerable interest from an information-theoretic perspective in the use of physical layer mechanisms to improve the security of wireless transmissions. Early work in this area was performed by Wyner, who introduced and studied the wiretap channel where the eavesdropper's received signal is a degraded version of the legitimate receiver's signal [1]. The non-degraded case was later studied by Csiszár and Körner [2].

Recently, many researchers have investigated secrecy in wiretap channels with multiple antennas [3]-[8]. In particular, for multiple-input single-output (MISO) wiretap channels, the optimal transmit covariance matrix was found to be singlestream beamforming obtained via a closed-form solution [4]. While research in this area usually assumes that global channel state information (CSI) is available at the transmitter, other work has considered the case where only partial information of the eavesdropper CSI (ECSI) is available. The optimal transmit covariance matrix that achieves the ergodic secrecy capacity for the MISO wiretap channel was studied in [5], where only statistical ECSI is assumed to be available. In [6]-[8], the

This work was supported by the U.S. Army Research Office under the Multi-University Research Initiative (MURI) grant W911NF-07-1-0318. problem was investigated from the perspective of maximizing the worst-case secrecy rate, where [6] considered the case that the eavesdropper's channel matrix is trace-bounded but otherwise unknown, and [7], [8] assumed that the channel mismatch is norm-bounded.

With the additional degrees of freedom available in multiantenna or multi-node systems, many papers have considered improving the secrecy rate through the use of artificial interference [3], [9], [10]. Recent work has also considered using friendly helpers to provide jamming signals to confuse the eavesdropper [11]-[13]. This approach is often referred to as cooperative jamming. The design of optimal transmit weights for multiple single-antenna helpers was studied in [11] with a global power constraint. In [13], the optimal beamforming strategy for a cooperative jammer was studied for the MISO case under a zero-forcing constraint that nulls the interference at the legitimate receiver. However, most of the previous work on cooperative jamming assumes perfect global channel state information, including CSI for the eavesdropper. This motivates us to investigate the case when the transmitters (including helpers) have only imperfect ECSI.

In this paper, we study robust transmit beamforming design for MISO wiretap channels with a helper. We assume that perfect CSI for the links to the legitimate user is available at both transmitters, while for the eavesdropper links there exist channel mismatches that are norm-bounded by some known constants [7], [14]. Following [4], Gaussian inputs are assumed in the paper. We focus on obtaining robust transmit covariance matrices (from which beamformers can be obtained via matrix decomposition) for the cooperative jamming scheme with a helper, based on satisfying certain quality-of-service (QoS) constraints at either the intended or malicious receiver, or both. Note that our work is different from [7] in that we consider robust transmission for not only information signals but also jamming signals. This paper is also different from our prior work in [14] which focused on maximizing the worst-case secrecy rate; here we work directly with SINR, since the ratio between the SINRs at the intended receiver and the eavesdropper can measure the secrecy performance of wiretap channels [9], [10]. We show that the non-convex problems for optimizing the worst-case secrecy performance can be converted into quasiconvex problems, and thus can be efficiently solved via existing methods.

The organization of the paper is as follows. Section II 
describes the system model considered throughout the paper. In Section III, robust design of the transmit covariance matrices are studied for various QoS-based scenarios. The performance of the proposed robust transmission approaches are studied using several simulation examples in Section IV, and conclusions are drawn in Section V.

The following notation is used in the paper: $\mathbb{E}\{\cdot\}$ denotes expectation, $(\cdot)^{H}$ the Hermitian transpose, $\|\cdot\|$ the Euclidean norm, $\operatorname{tr}(\cdot)$ is the trace operator, $[x]^{+}$represents $\max \{x, 0\}$, and $\mathbf{I}$ is an identity matrix of appropriate dimension.

\section{Mathematical Model}

We consider a MISO communication system with a source node (Alice), a helper (Helper), a destination (Bob), and an eavesdropper (Eve), as shown in Fig. 1. The number of antennas possessed by Alice and the Helper are denoted by $N_{a}$ and $N_{h}$, respectively, while both Bob and Eve are singleantenna nodes. In this model, Alice sends private messages to Bob in the presence of Eve, who is able to eavesdrop on the link between Alice and Bob. The Helper transmits artificial interference signals to confuse Eve. We assume that Alice and the Helper have perfect CSI for their links to Bob, but they have only imperfect CSI for their channels to Eve.

\section{A. Signal Model}

When Alice and the Helper transmit both information and jamming signals, the received signals at Bob and Eve are respectively given by

$$
\begin{aligned}
& y_{b}=\mathbf{h}_{b} \mathbf{x}+\mathbf{g}_{b} \mathbf{z}+n_{b} \\
& y_{e}=\mathbf{h}_{e} \mathbf{x}+\mathbf{g}_{e} \mathbf{z}+n_{e}
\end{aligned}
$$

where $\mathbf{x}$ is the signal vector transmitted by Alice, $\mathbf{z}$ is the i.i.d. Gaussian interference signal from the Helper, and $\left\{\mathbf{h}_{b}, \mathbf{h}_{e}\right\}$ are the $1 \times N_{a}$ channel vectors for Bob and Eve, respectively. The terms $n_{b}$ and $n_{e}$ represent naturally occurring noise at Bob and Eve, and we assume that $n_{b}$ and $n_{e}$ are zero-mean circular complex Gaussian with variance $\sigma_{b}^{2}$ and $\sigma_{e}^{2}$. We will assume without loss of generality that $\sigma_{b}^{2}=\sigma_{e}^{2}=\sigma^{2}$. We will let $\mathbf{Q}_{x}=\mathbb{E}\left\{\mathbf{x x}^{H}\right\}$ and $\mathbf{Q}_{z}=\mathbb{E}\left\{\mathbf{z z}^{H}\right\}$ denote the covariance matrices of the transmitted and information and jamming signals. While the robust beamforming optimization problems considered in the paper are subject to a global power constraint

$$
\operatorname{tr}\left(\mathbf{Q}_{x}\right)+\operatorname{tr}\left(\mathbf{Q}_{z}\right) \leq P,
$$

the generalization of the proposed solution to the case of individual power constraints $\operatorname{tr}\left(\mathbf{Q}_{x}\right) \leq P_{S}, \operatorname{tr}\left(\mathbf{Q}_{z}\right) \leq P_{J}$ is straightforward.

\section{B. Channel Mismatch}

For the channels between the transmitters and Eve, only estimates $\tilde{\mathbf{h}}_{e}$ and $\tilde{\mathbf{g}}_{e}$ are available at Alice and the Helper, respectively. We define the channel error vectors as

$$
\begin{aligned}
& \mathbf{e}_{h}=\mathbf{h}_{e}-\tilde{\mathbf{h}}_{e} \\
& \mathbf{e}_{g}=\mathbf{g}_{e}-\tilde{\mathbf{g}}_{e},
\end{aligned}
$$

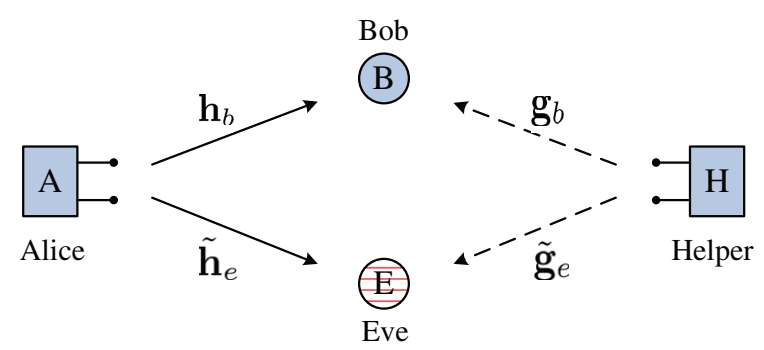

Fig. 1. System model.

and we assume that the channel mismatches lie in the bounded sets $\mathcal{E}_{h}=\left\{\mathbf{e}_{h}:\left\|\mathbf{e}_{h}\right\|^{2} \leq \epsilon_{h}^{2}\right\}$ and $\mathcal{E}_{g}=\left\{\mathbf{e}_{g}:\left\|\mathbf{e}_{g}\right\|^{2} \leq \epsilon_{g}^{2}\right\}$, where $\epsilon_{h}$ and $\epsilon_{g}$ are known constants.

\section{Performance Metric}

The secrecy rate of the above system as a function of $\mathbf{Q}_{x}$ and $\mathbf{Q}_{z}$ is given by [13]:

$$
R_{s}\left(\mathbf{Q}_{x}, \mathbf{Q}_{z}\right)=\left[\log _{2}\left(1+\Gamma_{b}\right)-\log _{2}\left(1+\Gamma_{e}\right)\right]^{+}
$$

where $\Gamma_{b}$ and $\Gamma_{e}$ are the SINRs at Bob and Eve, respectively:

$$
\begin{aligned}
\Gamma_{b} & =\frac{\mathbf{h}_{b} \mathbf{Q}_{x} \mathbf{h}_{b}^{H}}{\mathbf{g}_{b} \mathbf{Q}_{z} \mathbf{g}_{b}^{H}+\sigma^{2}} \\
\Gamma_{e} & =\frac{\left(\tilde{\mathbf{h}}_{e}+\mathbf{e}_{h}\right) \mathbf{Q}_{x}\left(\tilde{\mathbf{h}}_{e}+\mathbf{e}_{h}\right)^{H}}{\left(\tilde{\mathbf{g}}_{e}+\mathbf{e}_{g}\right) \mathbf{Q}_{z}\left(\tilde{\mathbf{g}}_{e}+\mathbf{e}_{g}\right)^{H}+\sigma^{2}} .
\end{aligned}
$$

Our goal is to design robust $\mathbf{Q}_{x}$ and $\mathbf{Q}_{z}$ to improve the secrecy performance in the network, based on the values for $\mathbf{e}_{h}^{*} \in \mathcal{E}_{h}$ and $\mathbf{e}_{g}^{*} \in \mathcal{E}_{g}$ that give the worst performance (i.e. the best SINR for Eve). Note that the robust optimal solutions for $\mathbf{Q}_{x}$ found in this paper are guaranteed to be unit-rank, even without the imposition of rank constraints on the optimization problems. Thus, the term "beamforming" is appropriate here, and the corresponding beamformer is determined by simply decomposing the solution found for $\mathbf{Q}_{x}$. On the other hand, there is no need for the covariance of the jamming signal to be rank one, since this signal is simply noise and will not be decoded. Note also that the QoS constraints we will consider are based on the SINR at Bob and/or Eve, so we will use the terms "SINR" and "QoS" interchangeably in the sequel.

\section{Robust CoOperative JAMming}

In this section, we consider the following worst-case optimization problems under imperfect ECSI: (a) minimizing $\Gamma_{e}$ while maintaining a QoS constraint $\Gamma_{b} \geq \gamma_{b}$ at Bob, (b) maximizing $\Gamma_{b}$ while satisfying a QoS constraint $\Gamma_{e} \leq \gamma_{e}$ at Eve, and (c) minimizing the global transmit power $\operatorname{tr}\left(\mathbf{Q}_{x}\right)+\operatorname{tr}\left(\mathbf{Q}_{z}\right)$ while satisfying certain SINR constraints at both Bob and Eve. These problems focus on different security performance goals. Problem (a) focuses on the reliability of the legitimate link, problem (b) aims to suppress the eavesdropping abilities to a certain level, and problem (c) guarantees a lower bound on the worst-case secrecy rate using minimum power resources. 


\section{A. QoS Constraint at Legitimate Node}

For the case where a QoS constraint is set for Bob, minimization of the worst-case SINR $\Gamma_{e}$ at Eve is addressed by

$$
\begin{aligned}
& \min _{\mathbf{Q}_{x} \succeq 0, \mathbf{Q}_{z} \succeq 0} \max _{\mathbf{e}_{h} \in \mathcal{E}_{h}, \mathbf{e}_{g} \in \mathcal{E}_{g}} \frac{\left(\tilde{\mathbf{h}}_{e}+\mathbf{e}_{h}\right) \mathbf{Q}_{x}\left(\tilde{\mathbf{h}}_{e}+\mathbf{e}_{h}\right)^{H}}{\left(\tilde{\mathbf{g}}_{e}+\mathbf{e}_{g}\right) \mathbf{Q}_{z}\left(\tilde{\mathbf{g}}_{e}+\mathbf{e}_{g}\right)^{H}+\sigma^{2}} \\
& \text { s.t. } \operatorname{tr}\left(\mathbf{Q}_{x}\right)+\operatorname{tr}\left(\mathbf{Q}_{z}\right) \leq P \\
& \frac{\mathbf{h}_{b} \mathbf{Q}_{x} \mathbf{h}_{b}^{H}}{\mathbf{g}_{b} \mathbf{Q}_{z} \mathbf{g}_{b}^{H}+\sigma^{2}} \geq \gamma_{b} .
\end{aligned}
$$

The difficulty in solving this problem comes from the nonlinear form of the objective function and the inner maximization over the channel mismatches. The inner maximization of the numerator in (6) is actually a non-convex problem. Therefore, we convert problem (6) into a solvable quasiconvex problem via the following proposition.

Proposition 1: Problem (6) is equivalent to the following problem

$$
\begin{aligned}
\min _{\mathbf{Q}_{x}, \mathbf{Q}_{z}, \mathbf{\Psi}, \mathbf{\Phi}, \mu, \nu} & \frac{\mu \epsilon_{h}^{2}+\operatorname{tr}\left[\left(\mathbf{Q}_{x}+\mathbf{\Psi}\right) \tilde{\mathbf{h}}_{e}^{H} \tilde{\mathbf{h}}_{e}\right]}{\sigma^{2}-\nu \epsilon_{g}^{2}+\operatorname{tr}\left[\left(\mathbf{Q}_{z}-\mathbf{\Phi}\right) \tilde{\mathbf{g}}_{e}^{H} \tilde{\mathbf{g}}_{e}\right]} \\
\text { s.t. } \quad & {\left[\begin{array}{cc}
\mathbf{\Psi} & \mathbf{Q}_{x} \\
\mathbf{Q}_{x} & \mu \mathbf{I}_{N_{a}}-\mathbf{Q}_{x}
\end{array}\right] \succeq 0 } \\
& {\left[\begin{array}{cc}
\mathbf{\Phi} & \mathbf{Q}_{z} \\
\mathbf{Q}_{z} & \nu \mathbf{I}_{N_{h}}+\mathbf{Q}_{z}
\end{array}\right] \succeq 0 } \\
& \operatorname{tr}\left(\mathbf{Q}_{x}\right)+\operatorname{tr}\left(\mathbf{Q}_{z}\right) \leq P \\
& \mathbf{Q}_{x} \succeq 0, \mathbf{Q}_{z} \succeq 0, \mu \geq 0, \nu \geq 0 \\
& \operatorname{tr}\left(\mathbf{Q}_{x} \mathbf{h}_{b}^{H} \mathbf{h}_{b}\right) \geq \gamma_{b}\left(\operatorname{tr}\left(\mathbf{Q}_{z} \mathbf{g}_{b}^{H} \mathbf{g}_{b}\right)+\sigma^{2}\right) .
\end{aligned}
$$

Proof: The maximin problem (6) can be transformed to

$$
\begin{gathered}
\min _{\mathbf{Q}_{x} \succeq 0, \mathbf{Q}_{z} \succeq 0, u \geq 0, t>0} \quad \frac{u}{t} \\
\text { s.t. } \quad\left(\tilde{\mathbf{h}}_{e}+\mathbf{e}_{h}\right) \mathbf{Q}_{x}\left(\tilde{\mathbf{h}}_{e}+\mathbf{e}_{h}\right)^{H} \leq u, \forall \mathbf{e}_{h}:\left\|\mathbf{e}_{h}\right\| \leq \epsilon_{h} \\
\sigma^{2}+\left(\tilde{\mathbf{g}}_{e}+\mathbf{e}_{g}\right) \mathbf{Q}_{z}\left(\tilde{\mathbf{g}}_{e}+\mathbf{e}_{g}\right)^{H} \geq t, \forall \mathbf{e}_{g}:\left\|\mathbf{e}_{g}\right\| \leq \epsilon_{g} \\
\operatorname{tr}\left(\mathbf{Q}_{x}\right)+\operatorname{tr}\left(\mathbf{Q}_{z}\right) \leq P \\
\mathbf{h}_{b} \mathbf{Q}_{x} \mathbf{h}_{b}^{H} \geq \gamma_{b}\left(\mathbf{g}_{b} \mathbf{Q}_{z} \mathbf{g}_{b}^{H}+\sigma^{2}\right) .
\end{gathered}
$$

Using the $\mathcal{S}$-procedure [15] for constraint (8b), we know that there exists an $\mathbf{e}_{h} \in \mathbb{C}^{N_{a}}$ satisfying both of the inequalities if and only if there exists a $\mu \geq 0$ such that

$$
\left[\begin{array}{cc}
\mu \mathbf{I}_{N_{a}}-\mathbf{Q}_{x} & -\mathbf{Q}_{x} \tilde{\mathbf{h}}_{e}^{H} \\
-\tilde{\mathbf{h}}_{e} \mathbf{Q}_{x} & -\tilde{\mathbf{h}}_{e} \mathbf{Q}_{x} \tilde{\mathbf{h}}_{e}^{H}-\sigma^{2}-\mu \epsilon_{h}^{2}+u
\end{array}\right] \succeq 0 .
$$

Then we can use the property of the generalized Schur complement [16] and rewrite (9) as

$$
\sigma^{2}+\mu \epsilon_{h}^{2}+\tilde{\mathbf{h}}_{e} \mathbf{Q}_{x} \tilde{\mathbf{h}}_{e}^{H}+\tilde{\mathbf{h}}_{e} \mathbf{Q}_{x}\left(\mu \mathbf{I}_{N_{a}}-\mathbf{Q}_{x}\right)^{\dagger} \mathbf{Q}_{x} \tilde{\mathbf{h}}_{e}^{H} \leq u .
$$

Similarly for constraints (8c), we use the same procedure and obtain

$$
-\nu \epsilon_{g}^{2}+\tilde{\mathbf{g}}_{e} \mathbf{Q}_{z} \tilde{\mathbf{g}}_{e}^{H}-\tilde{\mathbf{g}}_{e} \mathbf{Q}_{z}\left(\nu \mathbf{I}_{N_{h}}+\mathbf{Q}_{z}\right)^{\dagger} \mathbf{Q}_{z} \tilde{\mathbf{g}}_{e}^{H} \geq t
$$

where $\nu \geq 0$. Therefore, the problem in (8) can be rewritten as

$$
\begin{aligned}
\min _{\mathbf{Q}_{x}, \mathbf{Q}_{z}, \mu, \nu} & \frac{\mu \epsilon_{h}^{2}+\tilde{\mathbf{h}}_{e} \mathbf{Q}_{x} \tilde{\mathbf{h}}_{e}^{H}+\tilde{\mathbf{h}}_{e} \mathbf{Q}_{x}\left(\mu \mathbf{I}_{N_{a}}-\mathbf{Q}_{x}\right)^{\dagger} \mathbf{Q}_{x} \tilde{\mathbf{h}}_{e}^{H}}{\sigma^{2}-\nu \epsilon_{g}^{2}+\tilde{\mathbf{g}}_{e} \mathbf{Q}_{z} \tilde{\mathbf{g}}_{e}^{H}-\tilde{\mathbf{g}}_{e} \mathbf{Q}_{z}\left(\nu \mathbf{I}_{N_{h}}+\mathbf{Q}_{z}\right)^{\dagger} \mathbf{Q}_{z} \tilde{\mathbf{g}}_{e}^{H}} \\
\text { s.t. } & \operatorname{tr}\left(\mathbf{Q}_{x}\right)+\operatorname{tr}\left(\mathbf{Q}_{z}\right) \leq P \\
& \mathbf{Q}_{x} \succeq 0, \mathbf{Q}_{z} \succeq 0, \mu \geq 0, \nu \geq 0,
\end{aligned}
$$

which is equivalent to the following expression obtained by using auxiliary semidefinite matrices $\boldsymbol{\Phi}$ and $\boldsymbol{\Psi}$ :

$$
\begin{aligned}
\min _{\mathbf{Q}_{x}, \mathbf{Q}_{z}, \mu, \nu, \Psi, \Phi} & \frac{\mu \epsilon_{h}^{2}+\tilde{\mathbf{h}}_{e} \mathbf{Q}_{x} \tilde{\mathbf{h}}_{e}^{H}+\tilde{\mathbf{h}}_{e} \mathbf{\Psi} \tilde{\mathbf{h}}_{e}^{H}}{\sigma^{2}-\nu \epsilon_{g}^{2}+\tilde{\mathbf{g}}_{e} \mathbf{Q}_{z} \tilde{\mathbf{g}}_{e}^{H}-\tilde{\mathbf{g}}_{e} \mathbf{\Phi} \tilde{\mathbf{g}}_{e}^{H}} \\
\text { s.t. } & \mathbf{Q}_{x}\left(\mu \mathbf{I}_{N_{a}}-\mathbf{Q}_{x}\right)^{\dagger} \mathbf{Q}_{x} \preceq \mathbf{\Psi} \\
& \mathbf{Q}_{z}\left(\mu \mathbf{I}_{N_{h}}+\mathbf{Q}_{z}\right)^{\dagger} \mathbf{Q}_{z} \preceq \mathbf{\Phi} \\
& \operatorname{tr}\left(\mathbf{Q}_{x}\right)+\operatorname{tr}\left(\mathbf{Q}_{z}\right) \leq P \\
& \mathbf{Q}_{x} \succeq 0, \mathbf{Q}_{z} \succeq 0, \mu \geq 0, \nu \geq 0 .
\end{aligned}
$$

Using the Schur complement for constraints (12a) and (12b), the expression in (7) can then be obtained.

Note that problem (7) is a semidefinite program (SDP) with a non-negative linear fractional objective function (thus quasiconvex), a set of linear matrix inequalities (LMIs) and affine inequalities. Thus it can be solved via the bisection (using interior-point methods for each feasibility problem) [15].

\section{B. QoS Constraint at Eavesdropper}

Now we consider the scenario where an SINR constraint $\gamma_{e}$ is imposed at Eve, and the maximization of $\Gamma_{b}$ can be formulated as:

$$
\begin{array}{cl} 
& \max _{\mathbf{Q}_{x} \succeq 0, \mathbf{Q}_{z} \succeq 0} \\
\text { s.t. } \max _{\mathbf{e}_{h} \in \mathcal{E}_{h}, \mathbf{e}_{g} \in \mathcal{E}_{g}} & \frac{\mathbf{h}_{b} \mathbf{Q}_{x} \mathbf{h}_{b}^{H}}{\mathbf{g}_{b} \mathbf{Q}_{z} \mathbf{g}_{b}^{H}+\sigma^{2}} \\
& \frac{\left(\tilde{\mathbf{h}}_{e}+\mathbf{e}_{h}\right) \mathbf{Q}_{x}\left(\tilde{\mathbf{h}}_{e}+\mathbf{e}_{h}\right)^{H}}{\left(\tilde{\mathbf{g}}_{e}+\mathbf{e}_{g}\right) \mathbf{Q}_{z}\left(\tilde{\mathbf{g}}_{e}+\mathbf{e}_{g}\right)^{H}+\sigma^{2}} \leq \gamma_{e} \\
\operatorname{tr}\left(\mathbf{Q}_{x}\right)+\operatorname{tr}\left(\mathbf{Q}_{z}\right) \leq P .
\end{array}
$$

In this problem, a lower $\gamma_{e}$ means a more strict secrecy constraint for the transmission, which usually requires more power consumption for the jamming signals and less for the information data. Therefore, under a total power constraint (13c), Bob's received SINR will also be degraded, as will be discussed in Section IV. In order to solve problem (13), we give the following proposition.

Proposition 2: Problem (13) is equivalent to

$$
\begin{aligned}
\max _{\mathbf{Q}_{x}, \mathbf{Q}_{z}, \mu, \nu, u, t} & \frac{\mathbf{h}_{b} \mathbf{Q}_{x} \mathbf{h}_{b}^{H}}{\mathbf{g}_{b} \mathbf{Q}_{z} \mathbf{g}_{b}^{H}+\sigma^{2}} \\
\text { s.t. } & \mu \epsilon_{h}^{2}+\operatorname{tr}\left[\left(\mathbf{Q}_{x}+\mathbf{\Psi}\right) \tilde{\mathbf{h}}_{e}^{H} \tilde{\mathbf{h}}_{e}\right] \leq u \\
& \sigma^{2}-\nu \epsilon_{g}^{2}+\operatorname{tr}\left[\left(\mathbf{Q}_{z}-\mathbf{\Phi}\right) \tilde{\mathbf{g}}_{e}^{H} \tilde{\mathbf{g}}_{e}\right] \geq t \\
& {\left[\begin{array}{cc}
\mathbf{\Psi} & \mathbf{Q}_{x} \\
\mathbf{Q}_{x} & \mu \mathbf{I}_{N_{a}}-\mathbf{Q}_{x}
\end{array}\right] \succeq 0 } \\
& {\left[\begin{array}{cc}
\mathbf{\Phi} & \mathbf{Q}_{z} \\
\mathbf{Q}_{z} & \nu \mathbf{I}_{N_{h}}+\mathbf{Q}_{z}
\end{array}\right] \succeq 0 } \\
& \operatorname{tr}\left(\mathbf{Q}_{x}\right)+\operatorname{tr}\left(\mathbf{Q}_{z}\right) \leq P
\end{aligned}
$$




$$
\begin{aligned}
& \mathbf{Q}_{x} \succeq 0, \mathbf{Q}_{z} \succeq 0,\{\mu, \nu, u, t\} \geq 0 \\
& t \gamma_{e}-u \geq 0 .
\end{aligned}
$$

Proof: The proof is similar to the proof for Proposition 1 and the details are omitted here. As an outline, we can first rewrite constraint (13b) as

$$
\begin{aligned}
& \left(\tilde{\mathbf{h}}_{e}+\mathbf{e}_{h}\right) \mathbf{Q}_{x}\left(\tilde{\mathbf{h}}_{e}+\mathbf{e}_{h}\right)^{H} \leq u, \forall \mathbf{e}_{h}:\left\|\mathbf{e}_{h}\right\| \leq \epsilon_{h} \\
& \left(\tilde{\mathbf{g}}_{e}+\mathbf{e}_{g}\right) \mathbf{Q}_{z}\left(\tilde{\mathbf{g}}_{e}+\mathbf{e}_{g}\right)^{H}+\sigma^{2} \geq t, \forall \mathbf{e}_{g}:\left\|\mathbf{e}_{g}\right\| \leq \epsilon_{g} \\
& \frac{u}{t} \leq \gamma_{e} .
\end{aligned}
$$

Then we can use the same procedure as in (8)-(11) on constraints (15)-(16), and the desired conclusion can be obtained.

Note that problem (14) is also an SDP with a quasiconvex objective function, and thus can be solved with bisection method, similar to (7).

\section{QoS Constraint at Both Receivers}

Here we consider a slightly different problem in which there are QoS constraints at both Bob and Eve. The problem of minimizing the global transmit power under these constraints is formulated as

$$
\begin{aligned}
& \min _{\mathbf{Q}_{x} \succeq 0, \mathbf{Q}_{z} \succeq 0} \operatorname{tr}\left(\mathbf{Q}_{x}\right)+\operatorname{tr}\left(\mathbf{Q}_{z}\right) \\
& \text { s.t. } \max _{\mathbf{e}_{h} \in \mathcal{E}_{h}, \mathbf{e}_{g} \in \mathcal{E}_{g}} \frac{\left(\tilde{\mathbf{h}}_{e}+\mathbf{e}_{h}\right) \mathbf{Q}_{x}\left(\tilde{\mathbf{h}}_{e}+\mathbf{e}_{h}\right)^{H}}{\left(\tilde{\mathbf{g}}_{e}+\mathbf{e}_{g}\right) \mathbf{Q}_{z}\left(\tilde{\mathbf{g}}_{e}+\mathbf{e}_{g}\right)^{H}+\sigma^{2}} \leq \gamma_{e} \\
& \\
& \frac{\mathbf{h}_{b} \mathbf{Q}_{x} \mathbf{h}_{b}^{H}}{\mathbf{g}_{b} \mathbf{Q}_{z} \mathbf{g}_{b}^{H}+\sigma^{2}} \geq \gamma_{b} .
\end{aligned}
$$

Proposition 3: Problem (18) is equivalent to the following problem

$$
\begin{aligned}
\min _{\mathbf{Q}_{x}, \mathbf{Q}_{z}, \mu, \nu, u, t} & \operatorname{tr}\left(\mathbf{Q}_{x}\right)+\operatorname{tr}\left(\mathbf{Q}_{z}\right) \\
\text { s.t. } & \mu \epsilon_{h}^{2}+\operatorname{tr}\left[\left(\mathbf{Q}_{x}+\mathbf{\Psi}\right) \tilde{\mathbf{h}}_{e}^{H} \tilde{\mathbf{h}}_{e}\right] \leq u \\
& \sigma^{2}-\nu \epsilon_{g}^{2}+\operatorname{tr}\left[\left(\mathbf{Q}_{z}-\mathbf{\Phi}\right) \tilde{\mathbf{g}}_{e}^{H} \tilde{\mathbf{g}}_{e}\right] \geq t \\
& {\left[\begin{array}{cc}
\mathbf{\Psi} & \mathbf{Q}_{x} \\
\mathbf{Q} & \mu \mathbf{I}_{N_{a}}-\mathbf{Q}_{x}
\end{array}\right] \succeq 0 } \\
& {\left[\begin{array}{cc}
\mathbf{\Phi} & \mathbf{Q}_{z} \\
\mathbf{Q}_{z} & \nu \mathbf{I}_{N_{h}}+\mathbf{Q}_{z}
\end{array}\right] \succeq 0 } \\
& \operatorname{tr}\left(\mathbf{Q}_{x} \mathbf{h}_{b}^{H} \mathbf{h}_{b} \geq \gamma_{b}\left(\operatorname{tr}\left(\mathbf{Q}_{z} \mathbf{g}_{b}^{H} \mathbf{g}_{b}\right)+\sigma^{2}\right)\right. \\
& \mathbf{Q}_{x} \succeq 0, \mathbf{Q}_{z} \succeq 0,\{\mu, \nu, u, t\} \geq 0 \\
& t \gamma_{e}-u \geq 0 .
\end{aligned}
$$

The proof is similar to that for the previous propositions and is thus omitted . Unlike (7) and (14), problem (19) only contains a linear objective function, so a bisection method is not needed in this case, and we can solve this SDP more efficiently. Also note that $\gamma_{b}$ and $\gamma_{e}$ in (19) implicitly indicate a guaranteed secrecy rate; that is, the worst-case secrecy rate will not be lower than $\left[\log _{2}\left(1+\gamma_{b}\right)-\log _{2}\left(1+\gamma_{e}\right)\right]^{+}$.

Note that by examining the Karush-Kuhn-Tucker (KKT) conditions for the primary optimization problems (6), (13) and (18) in this paper, we can also prove (omitted due to space limitations) that given any jamming covariance $\mathbf{Q}_{z}$ and

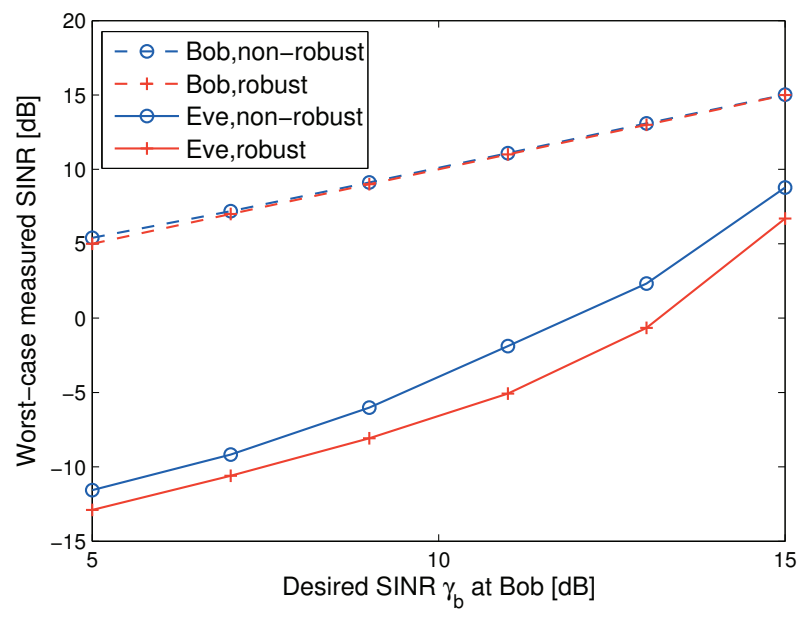

Fig. 2. Worst-case measured SINR vs. desired SINR at Bob, $\epsilon_{h}^{2}=\epsilon_{g}^{2}=0.5$, $P=10 \mathrm{~dB}$.

channel mismatches $\mathbf{e}_{h}$ and $\mathbf{e}_{g}$, the solution for the information covariance $\mathbf{Q}_{x}$ is always unit-rank. Therefore, single-stream beamformers at Alice can be obtained accordingly.

\section{Numerical Results}

In this section, we present some numerical examples on the secrecy rate performance of the robust transmission schemes studied in the paper. For all examples, we assume Alice and the Helper both have four antennas, i.e. $N_{a}=N_{h}=4$, while Bob and Eve each has one. The channel matrices are assumed to be composed of independent, zero-mean Gaussian random variables with unit variance. All results are calculated based on an average of 1000 independent trials. The background noise power is assumed to be the same at Bob and Eve, $\sigma_{b}^{2}=\sigma_{e}^{2}=$ 1 , and the transmit power $P$ is defined in $\mathrm{dB}$ relative to the noise power.

We will examine the performance of the proposed robust cooperative jamming schemes for the QoS-based scenarios discussed in Section III. For purpose of comparison, we also simulate the non-robust counterparts of these schemes, by dropping the consideration for channel mismatches $\epsilon_{h}$ and $\epsilon_{g}$ and using only the channel estimates $\tilde{\mathbf{h}}_{e}$ and $\tilde{\mathrm{g}}_{e}$ for Eve's channel. The channel mismatch bounds are assumed to be $\epsilon_{h}^{2}=\epsilon_{g}^{2}=0.5$ for all the examples.

In Fig. 2, we consider the case where a desired SINR constraint $\gamma_{b}$ is imposed at Bob, $P$ is assumed to be $10 \mathrm{~dB}$, and we plot the measured SINR at Bob and Eve with an increasing SINR constraint at Bob. Since no channel errors are assumed for Bob's channel, the SINR constraint is met in all cases and both curves for Bob coincide. We see that the robust cooperative jamming scheme provides several $\mathrm{dB}$ of improvement in suppressing the worst-case SINR at Eve. When the SINR requirement at Bob is higher, more power will be allocated to Alice and less power will be available for jamming. Therefore, the SINR at Eve also increases and hence the secrecy is reduced. 


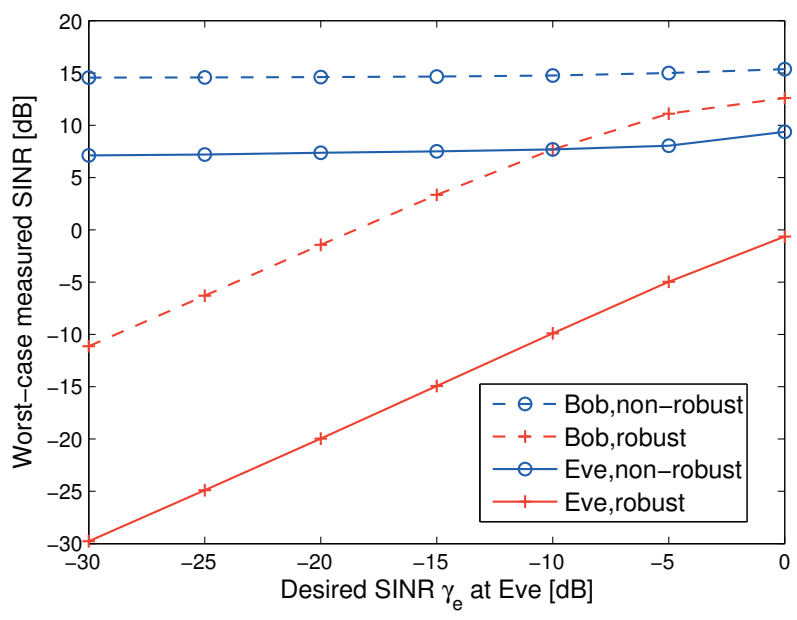

Fig. 3. Worst-case measured SINR vs. desired SINR at Eve, $\epsilon_{h}^{2}=\epsilon_{g}^{2}=0.5$, $P=10 \mathrm{~dB}$.

The performance gain of the robust design is more obvious in Fig. 3, where the SINR constraint is set at Eve with a global power limit of $10 \mathrm{~dB}$, and $\gamma_{e}$ ranges from $-30 \mathrm{~dB}$ to $0 \mathrm{~dB}$. It can be seen that the robust scheme guarantees that the constraint is met for the eavesdropper link (i.e. the SINR at Eve remains equal to or lower than required). However, for the non-robust scheme, the Eve's actual SINR exceeds the required threshold by at least $10 \mathrm{~dB}$ (as high as $35 \mathrm{~dB}$ in the range of interest) due to the impact of channel error. In addition, the ratio between the SINRs at Bob and Eve (which can roughly measure the secrecy performance) is also higher for the robust scheme, compared to that for the non-robust scheme.

The SINR performance and minimum required transmit power for satisfying QoS constraints at both Bob and Eve are shown in Fig. 4, where $\gamma_{e}$ is fixed at $-10 \mathrm{~dB}$ and $\gamma_{b}$ ranges from $5 \mathrm{~dB}$ to $15 \mathrm{~dB}$. We see that the use of robust cooperative jamming can satisfy both the SINR requirements at Bob and Eve, while its non-robust counterpart can only meet the demand for Bob and produces much higher information leakage to Eve. We can also see from the transmit power plot that although the robustness and higher secrecy performance introduced by the robust beamforming requires higher transmit power, the improvement in the secrecy performance is higher than the power increase and grows with larger $\gamma_{b}$.

\section{CONCLUSions}

In this paper, we studied robust beamforming designs for MISO wiretap channels with a helper, under the assumption that the CSIs for eavesdropper's links are imperfect. Robust transmit covariance matrices were obtained for various QoSconstrained scenarios, based on the worst-case performance optimization. We showed that the original non-convex optimization problems for robust design can be transformed to quasiconvex problems, and thus can be efficiently solved with existing methods. The benefits of the robust designs were illustrated through numerical results.
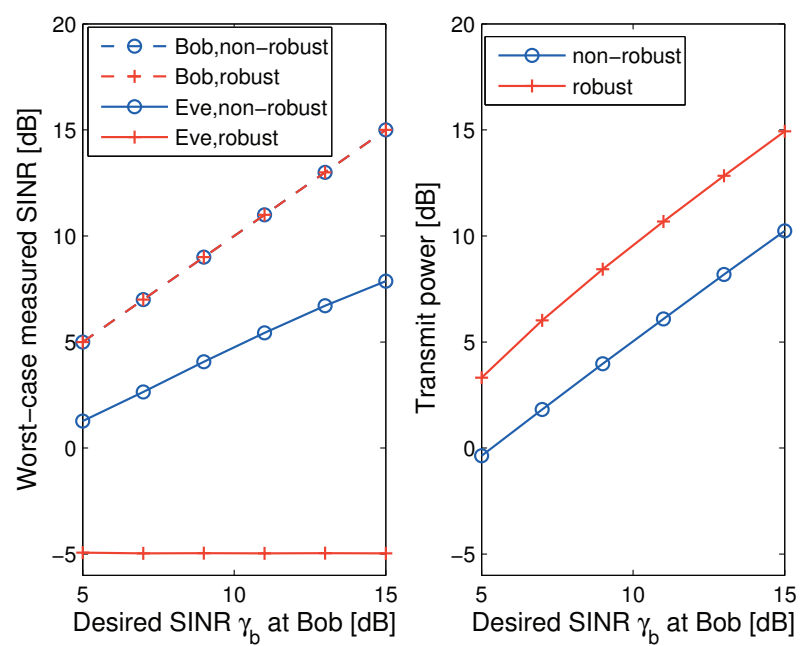

Fig. 4. Worst-case measured SINR and transmit power vs. desired SINR at Bob, $\epsilon_{h}^{2}=\epsilon_{g}^{2}=0.5, \gamma_{e}=-5 \mathrm{~dB}$.

\section{REFERENCES}

[1] A. D. Wyner, "The wire-tap channel," Bell System Technical Journal, vol. 54, no. 8, pp. 1355-1387, Jan. 1975.

[2] I. Csiszár and J. Körner, "Broadcast channels with confidential messages," IEEE Trans. Inf. Theory, vol. 24, no. 3, pp. 339-348, May 1978.

[3] S. Goel and R. Negi, "Guaranteeing secrecy using artificial noise," IEEE Trans. Wireless Commun., vol. 7, no. 6, pp. 2180-2189, Jun. 2008.

[4] S. Shafiee and S. Ulukus, "Achievable rates in Gaussian MISO channels with secrecy constraints," in Proc. IEEE ISIT, Jun. 2007, pp. 2466-2470.

[5] J. Li and A. P. Petropulu, "On ergodic secrecy rate for Gaussian MISO wiretap channels," IEEE Trans. Wireless Commun., vol. 10, no. 4, pp. 1176-1187, Apr. 2011.

[6] A. Wolf and E. Jorswieck, "Maximization of worst-case secrecy rates in MIMO wiretap channels," in Proc. 44th Asilomar Conference on Signals, Systems and Computers, Nov. 2010.

[7] L. Zhang, Y.-C. Liang, Y. Pei, and R. Zhang, "Robust beamforming design: From cognitive radio MISO channels to secrecy MISO channels," in Proc. IEEE GLOBECOM, Nov. 2009, pp. 1-5.

[8] Q. Li and W.-K. Ma, "Optimal and robust transmit designs for MISO channel secrecy by semidefinite programming," IEEE Trans. Signal Process., vol. 59, no. 8, pp. 3799-3812, Aug. 2011.

[9] A. L. Swindlehurst, "Fixed SINR solutions for the MIMO wiretap channel," in Proc. IEEE ICASSP, Apr. 2009, pp. 2437-2440.

[10] A. Mukherjee and A. L. Swindlehurst, "Robust beamforming for security in MIMO wiretap channels with imperfect CSI," IEEE Trans. Signal Process., vol. 59, no. 1, pp. 351-361, Jan. 2011.

[11] L. Dong, Z. Han, A. P. Petropulu, and H. V. Poor, "Improving wireless physical layer security via cooperating relays," IEEE Trans. Signal Process., vol. 58, no. 3, pp. 1875-1888, Mar. 2010.

[12] J. Huang and A. L. Swindlehurst, "Cooperative jamming for secure communications in MIMO relay networks," IEEE Trans. Signal Process., vol. 59 , no. 10 , pp. 4871-4884, Oct. 2011.

[13] A. Wolf and E. A. Jorswieck, "On the zero forcing optimality for friendly jamming in MISO wiretap channels," in Proc. IEEE SPAWC, Jun. 2010, pp. 1-5.

[14] J. Huang and A. L. Swindlehurst, "Robust secure transmission in MISO channels based on worst-case optimization," Apr. 2011. [Online]. Available: http://arxiv.org/abs/1104.3161

[15] S. P. Boyd and L. Vandenberghe, Convex Optimization. Cambridge University Press, 2004.

[16] D. Carlson, E. Haynsworth, and T. Markham, "A generalization of the Schur complement by means of the Moore-Penrose inverse," SIAM Journal on Applied Mathematics, vol. 26, no. 1, pp. 169-175, Jan. 1974. 